\title{
Carga de trabalho na enfermagem: variável do dimensionamento de pessoal
}

Recebido em: 30/06/2010

Aceito em: 12/11/2011
Márcia Elisa Binder Neis ${ }^{1}$ Francine Lima Gelbcke ${ }^{2}$

O objetivo deste estudo foi conhecer a produção teórica sobre carga de trabalho enquanto variável do dimensionamento de pessoal de enfermagem a partir da revisão da literatura em trabalhos na forma de artigos, teses ou dissertações, em português, indexados na base de dados BDTD e BVS, nos últimos dez anos. Foram selecionados 26 artigos de periódicos e 22 teses/dissertações. Os resultados evidenciaram a associação da carga de trabalho a quatro grupos: instrumentos para mensurar carga de trabalho, carga de trabalho em UTI, carga de trabalho e condições de trabalho e "outros", por serem de áreas bem diferentes.

Descritores: Carga de Trabalho, Enfermagem, Pessoal de Enfermagem.

\section{Workload in nursing work: variable dimensioning of nursing staff}

The aim of this study was to know the theoretical production on workload, while variable dimensioning of nursing staff, from a review of literature in papers in article format, thesis or dissertations, in Portuguese, indexed in the database DBTD and BVS, in the last ten years. Twenty-six journal articles and twentytwo dissertation/thesis have been selected. The results showed the association of the workload to four groups: instruments for measuring workload, workload in the ICU, workload and working conditions and "others" for being from very different areas.

Descriptors: Workload, Nursing, Nursing Staff.

\section{Carga de trabajo de enfermería: variable del dimensionamiento de enfermeras}

El objetivo de este estudio era entender la producción teórica sobre la carga de trabajo, mientras que la escala variable para dimensionamiento de enfermeras, en revisión de la literatura en forma de artículos, tesis o disertaciones, en portugués, indexada en la base de datos BDTD y BVS, en los últimos diez años. Se han seleccionado los 26 artículos de publicaciones periódicas y 22 tesis/disertaciones. Los resultados revelan la carga de trabajo de asociación a cuatro grupos: instrumentos para medir la carga de trabajo, carga de trabajo en UTI y las condiciones de trabajo, y "otro" por ser bastante diferentes áreas.

Descriptores: Carga de Trabajo, Enfermería, Personal de Enfermería.

\section{INTRODUÇÃO}

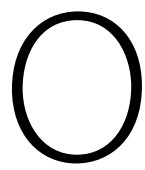

s profissionais são responsáveis pelo planejamento quantiqualitativo dos profissionais de enfermagem necessários à prestação da assistência de enfermagem, conforme a lei do exercício profissional 7498 de 26 de junho de $1986^{(1)}$ e a resolução do Conselho Federal de Enfermagem - Cofen 293/2004 ${ }^{(2)}$.

A previsão do quantitativo de pessoal de enfermagem é um processo que depende do conhecimento da carga de trabalho existente nas unidades de internação, que está relacionada às necessidades de assistência de enfermagem dos pacientes e do padrão de cuidado pretendido ${ }^{(3)}$.

Para a definição dos recursos humanos necessários às demandas do trabalho de enfermagem, há que se observar diferentes variáveis: atividades a serem realizadas, complexidade e necessidade de qualificação técnica dos profissionais, grau de dependência da clientela, tecnologia necessária para o desenvolvimento do trabalho, recursos técnicos e materiais disponíveis, além de características de ordem técnica, científica e pessoal dos trabalhadores, entre outras.
Para a definição do quantitativo de pessoal, Gaidzinski(3) desenvolveu uma equação que permite dimensionar o quadro de pessoal de enfermagem por complexidade assistencial, conforme os critérios estabelecidos pela resolução nº 189/1996 do Cofen ${ }^{(4-5)}$. Em sua aplicação, necessita da identificação das seguintes variáveis: carga de trabalho da unidade, índice de segurança técnica e tempo efetivo de trabalho.

Alguns autores defendem que a medida da carga de trabalho na enfermagem é um fator indispensável para o adequado provimento de pessoal em unidades hospitalares, devendo ser balizada pela demanda dos pacientes e pelos padrões de cuidados estabelecidos como meta pelas instituições ${ }^{(3-6)}$.

Define-se carga de trabalho da unidade de assistência de enfermagem como o produto da quantidade média diária de pacientes assistidos, segundo o grau de dependência dos cuidados de enfermagem, pelo tempo médio de assistência de enfermagem utilizado por paciente, de acordo com o grau de dependência apresentado, levantado através de um Sistema de Classificação de Pacientes (SCP) ${ }^{(3)}$.

1 Enfermeira do Centro de Esterilização do Hospital Universitário da Universidade Federal de Santa Catarina. Especialista em Administração Hospitalar e em Centro Cirúrgico. Mestranda do Programa de Pós-Graduação da Universidade Federal de Santa Catarina (UFSC). Membro do Grupo de Pesquisa Práxis da UFSC. E-mail: marcianeis@gmail.com. 2 Doutora em Enfermagem. Diretora de Enfermagem do Hospital Universitário/UFSC. Professora Adjunta do Departamento de Enfermagem/UFSC. Coordenadora do Curso de Mestrado Profissional em Gestão do Cuidado em Enfermagem do HU/UFSC. 
Nessa direção, o presente estudo buscou responder à seguinte questão: o que tem sido produzido na literatura nacional sobre carga de trabalho em enfermagem enquanto variável do dimensionamento de pessoal de enfermagem?

\section{METODOLOGIA}

Estudo caracterizado como revisão integrativa de literatura, seguiu modelo analítico ${ }^{(7)}$ que possibilitou o processo de pesquisa e síntese dos resultados de estudos independentes, no qual as informações foram categorizadas de forma sistemática. Essa revisão foi organizada a partir de um protocolo constando das etapas: definição da pergunta de pesquisa e dos critérios de seleção de estudos e amostra; representação da amostra em formato de tabela, considerando as características comuns; apresentação dos resultados identificando semelhanças e diferenças.

O levantamento bibliográfico foi realizado nas bases de dados da Biblioteca Virtual de Saúde (BVS) e do Banco Digital de Teses (BDTD), limitando-se às publicações dos últimos dez anos (de janeiro de 2000 a março de 2010), entre maio e junho de 2010, considerando as expressões "carga de trabalho" e "enfermagem".

Para a constituição da amostra, foram selecionados os trabalhos com textos disponíveis na íntegra que abordam a temática "carga de trabalho" em língua portuguesa.

Foram identificadas 128 publicações, sendo 84 na Lilacs e 44 na Scielo. Para a seleção dos estudos, após a leitura dos títulos e resumos, foi excluída toda publicação duplicada, não convergente ao escopo do estudo e não disponível na íntegra. Considerando o critério de pertinência e consistência de conteúdo, foram selecionadas 48 bibliografias, das quais 22 são teses/dissertações e 26, artigos científicos.

Para o processo de seleção, foi utilizado um formulário de análise individual dos estudos, contendo: referência, tipo de artigo (pesquisa, revisão de literatura ou outros), objetivo central do artigo, metodologia (tipo de estudo, sujeitos pesquisados, instrumentos de coleta de dados) e resultados principais do estudo. A partir da leitura dos trabalhos, os achados foram sistematizados e categorizados.

\section{PRODUÇÃO SOBRE CARGA DE TRABALHO-RESULTADOS}

A partir da coleta e análise prévia dos dados, esses foram agrupados em tabelas, conforme o tipo de material coletado: artigos (tabela 1) e teses e dissertações (tabela 2), apresentadas a seguir.

Tabela 1 - Distribuição dos estudos em periódicos segundo origem, ano e autor, no período de janeiro de 2000 a março de 2010

\begin{tabular}{|c|c|c|c|c|c|}
\hline Origem da publicação & Ano & Autores & Origem da publicação & Ano & Autores \\
\hline Rev. Baiana Saúde Pública & 2003 & Santos; Araújo & \multirow{3}{*}{ Rev. Latinoam. Enferm. } & \multirow[t]{3}{*}{2008} & Souza; Gonçalves; \\
\hline Rev. Uerj & 2005 & Farias; Zeitoune & & & Toffoletto; \\
\hline Rev. Bras. Enferm. & 2005 & Nicola; Anselmi & & & Leão; Padilha \\
\hline \multirow[t]{3}{*}{ Rev. Esc. Enferm. USP } & \multirow[t]{3}{*}{2005} & \multirow{3}{*}{$\begin{array}{l}\text { Padilha; Souza; } \\
\text { Miyadahira; Cruz; } \\
\text { Vattimo; Kimura; } \\
\text { Grossi; Silva; } \\
\text { Cruz; Ducci }\end{array}$} & Rev. Esc. Enferm. USP & 2008 & $\begin{array}{l}\text { Ducci; Zanei; } \\
\text { Whitaker }\end{array}$ \\
\hline & & & Acta Paul. Enferm. & 2008 & Ducci; Padilha \\
\hline & & & Texto \& Contexto Enferm. & 2008 & $\begin{array}{l}\text { Lima; Tsukamoto; } \\
\text { Fugulin }\end{array}$ \\
\hline Rev. Bras. Enferm. & 2006 & Gonçalves; Padilha & \multirow[t]{2}{*}{ Rev. Latinoam. Enferm. } & \multirow[t]{2}{*}{2009} & \multirow{2}{*}{$\begin{array}{l}\text { Balsanelli; Cunha; } \\
\text { Whitaker }\end{array}$} \\
\hline \multirow[t]{2}{*}{ Acta Paul. Enferm. } & \multirow[t]{2}{*}{2006} & \multirow{2}{*}{$\begin{array}{l}\text { Balsanelli; Zanei; } \\
\text { Whitaker }\end{array}$} & & & \\
\hline & & & \multirow[t]{2}{*}{ Rev. Bras. Enferm. } & \multirow[t]{2}{*}{2009} & \multirow{2}{*}{$\begin{array}{l}\text { Magalhães; Riboldi; } \\
\text { Dall'Agnol }\end{array}$} \\
\hline Rev. Esc. Enferm. USP & 2006 & Toffoletto; Padilha & & & \\
\hline Rev. Bras. Ter. Intensiva & 2006 & Dias; Matta; Nunes & \multirow[t]{2}{*}{ Texto \& Contexto Enferm. } & \multirow[t]{2}{*}{2009} & \multirow{2}{*}{$\begin{array}{l}\text { Corrente; Zornoff; } \\
\text { Arantes }\end{array}$} \\
\hline \multirow[t]{2}{*}{ Rev. Esc. Enferm. USP } & \multirow[t]{2}{*}{2007} & \multirow{2}{*}{$\begin{array}{l}\text { Dal Bem; } \\
\text { Gaidzinski }\end{array}$} & & & \\
\hline & & & Rev. Esc. Enferm. USP & 2009 & Queijo; Padilha \\
\hline \multirow[t]{2}{*}{ Rev. Bras. Epidemiol. } & \multirow[t]{2}{*}{2007} & $\begin{array}{l}\text { Gehring Junior; } \\
\text { Correa Filho; }\end{array}$ & Rev. Latinoam. Enferm. & 2009 & $\begin{array}{l}\text { Panunto; } \\
\text { Guirardello }\end{array}$ \\
\hline & & $\begin{array}{l}\text { Vieira Neto; } \\
\text { Ferreira; Vieira }\end{array}$ & \multirow[t]{2}{*}{ Rev. Esc. Enferm. USP } & \multirow[t]{2}{*}{2009} & $\begin{array}{l}\text { Souza; Padilha; } \\
\text { Nogueira; }\end{array}$ \\
\hline Rev. Bras. Enferm. & 2007 & Ribeiro; Shimizu & & & Miyadahira; Oliveira \\
\hline Rev. Esc. Enferm. USP & 2007 & Conishi; Gaidzinski & \multirow[t]{4}{*}{ Rev. Esc. Enferm. USP } & \multirow[t]{4}{*}{2009} & \multirow{4}{*}{$\begin{array}{l}\text { Gaidzinski; Fugulin; } \\
\text { Peres; Castilho; } \\
\text { Massarollo; } \\
\text { Mira; Pereira; } \\
\text { Tsukamoto }\end{array}$} \\
\hline Rev. Latinoam. Enferm. & 2007 & Telles; Castilho & & & \\
\hline Rev. Esc. Enferm. USP & 2007 & Gonçalves; Padilha & & & \\
\hline Cogitare Enferm. & 2007 & $\begin{array}{l}\text { Wolff; Mazur; } \\
\text { Wiezbicki; } \\
\text { Barros; Quadros }\end{array}$ & & & \\
\hline
\end{tabular}


Tabela 2 - Distribuição das produções de programas de pósgraduação segundo origem, ano e autor, no período de janeiro de 2000 a março de 2010

\begin{tabular}{|c|c|c|}
\hline Origem da publicação & Ano & Autores \\
\hline $\begin{array}{l}\text { Universidade de } \\
\text { São Paulo (USP) }\end{array}$ & 2000 & Berenice Nunes \\
\hline USP & 2000 & Márcia Perroca \\
\hline USP & 2002 & Gisela Nogueira \\
\hline USP & 2002 & Teresa Horibe \\
\hline USP & 2002 & Alda Queijo \\
\hline $\begin{array}{l}\text { Universidade do } \\
\text { Estado do Rio } \\
\text { de Janeiro (Uerj) }\end{array}$ & 2003 & Mônica Souto \\
\hline USP & 2004 & Marisol Carvalho \\
\hline $\begin{array}{l}\text { Universidade } \\
\text { Federal do Rio } \\
\text { Grande do Sul (UFRGS) }\end{array}$ & 2004 & Sílvia Piesanti \\
\hline UFRGS & 2005 & Cássia Costa \\
\hline Uerj & 2005 & $\begin{array}{l}\text { Fernando Henrique; } \\
\text { Brandão Molento }\end{array}$ \\
\hline USP & 2006 & Eliane Cruz \\
\hline USP & 2006 & Nanci Santos \\
\hline USP & 2006 & Márcia Dias \\
\hline USP & 2006 & lara Sêcco \\
\hline USP & 2006 & Leilane Gonçalves \\
\hline USP & 2007 & $\begin{array}{l}\text { Luciana } \\
\text { Bochembuzio }\end{array}$ \\
\hline USP & 2007 & Adriana Ducci \\
\hline $\begin{array}{l}\text { Faculdade de } \\
\text { Medicina de } \\
\text { Botucatu }\end{array}$ & 2008 & Meire Castro \\
\hline USP & 2008 & Denise Cheavegatt \\
\hline USP & 2008 & Alda Queijo \\
\hline USP & 2009 & Janaína Costa \\
\hline USP & 2009 & Alda Soares \\
\hline
\end{tabular}

\section{DISCUSSÕES}

A reflexão sobre cargas de trabalho como variável do dimensionamento de pessoal é anterior ao período pesquisado. Porém, observa-se que, a partir de 2005, há um aumento da produção científica, o que pode estar relacionado com a aprovação da resolução 293/2004 do Cofen, que fixa e estabelece parâmetros para o dimensionamento do quadro de profissionais de enfermagem nas unidades assistenciais das instituições de saúde e assemelhados. Os caminhos apontados a partir dessa resolução parecem ter incentivado os enfermeiros a se aventurarem nas pesquisas nesse campo de investigação.

As tabelas 1 e 2 espelham uma concentração da produção científica acerca dessa temática no estado de São Paulo. Acredita-se que esse fato possa estar relacionado à existência na Universidade de São Paulo (USP) de grupos de pesquisa cadastrados no Conselho Nacional de Desenvolvimento Científico e Tecnológico (CNPq), que têm estudado os vários aspectos do dimensionamento de pessoal de enfermagem e, consequentemente, a carga de trabalho.

Os dados organizados nas tabelas e a leitura flutuante dos mesmos possibilitou agrupá-los em quatro grandes categorias: instrumentos para mensurar carga de trabalho; carga de trabalho em UTI; carga de trabalho e condições de trabalho; e "outros", que foram assim denominados por abrangerem diversas áreas sem correlação com as categorias anteriores.

Os primeiros estudos desta última década foram focados na validação de instrumentos para mensurar carga de trabalho, constituindo a primeira grande categoria - instrumentos para mensurar carga de trabalho.

A pioneira foi Perroca, que, em sua tese de doutorado, monitorou a confiabilidade e a validade do instrumento de classificação de pacientes proposto por ela mesma em 1996, baseado nas necessidades individualizadas de cuidado de enfermagem, aplicado a UTI e Unidades de Internação(8). Estudos também foram realizados visando a validar os instrumentos Intervention Scoring System-28 (Tiss-28) ${ }^{(9)}$ e Nursing Activities Score (NAS) ${ }^{(10)}$. Outros autores comparam instrumentos como o Nine Equivalents of Nursing Manpower Score (Nems) e o Simplified Acute Physiology Score II (Saps II) com o NAS e o Tiss-28, a fim de verificar quais eram os mais adequados à realidade pesquisada ${ }^{(11-14)}$.

Das 48 literaturas analisadas, 16 eram relacionados à UTI. Nota-se que os instrumentos disponíveis para a avaliação da carga de trabalho estão relacionados a esse serviço, mas, além da disponibilidade de instrumentos, há que se considerar a complexidade de atendimento aos pacientes nesse serviço, gerando estudos sobre carga de trabalho em UTI.

As condições de trabalho apareceram interferindo na carga de trabalho, sendo que em vários estudos foram identificadas propostas para melhorá-las ${ }^{(15-17)}$. Esses estudos constituíram a categoria carga de trabalho e condições de trabalho.

Alguns estudos ${ }^{(18-19)}$ analisaram as condições de trabalho em UTIs, identificando a sobrecarga de trabalho e seus agentes causadores, sem propor soluções.

Autores concordam que as condições de trabalho inadequadas interferem na carga de trabalho dos funcionários e que essa "sobrecarga" causa problemas de saúde físicos e psíquicos. Um dos autores também levanta essa sobrecarga, sem associar a problemas de saúde, mas a questões relacionais e de processo de trabalho ${ }^{(18)}$.

Nessa mesma linha, outro autor ${ }^{(20)}$ relacionou carga de trabalho com condições de trabalho devido a falta de insumos, equipamentos de proteção individual e educação em serviço e incluiu o processo de trabalho como condicionante que eleva essa carga de trabalho.

No grupo "outros", foram enquadrados estudos que abordam a identificação de carga de trabalho em setores como alojamento conjunto, centro de esterilização, unidade de gastroenterologia, assistência domiciliar, além da aplicação dos instrumentos validados para UTI em setores como 
pediatria, por exemplo.

A análise dos dados e as categorias definidas apontam ainda uma lacuna no estudo acerca de carga de trabalho como variável do dimensionamento de pessoal a ser aplicada nas diversas áreas dos serviços de saúde e, em especial, na área hospitalar.

\section{CONSIDERAÇÕES FINAIS}

Os estudos apontam que o enfermeiro tem buscado instrumentos que lhe deem alguma objetividade para levantar a carga de trabalho em sua área de atuação.

Observa-se que vários sistemas de verificação de carga de trabalho na enfermagem foram criados na tentativa de avaliar o tipo de paciente atendido. Embora os critérios fossem diferentes em alguns aspectos (físicos, psicológicos, outros), todos eles trouxeram contribuição para a avaliação dos pacientes e de suas necessidades de cuidados pela equipe de enfermagem.

Acredita-se que os sistemas de escores aplicados na UTI, o setor mais pesquisado, têm se tornado também uma importante ferramenta para medir o desempenho na UTI, podendo ser utilizados para determinação da carga de trabalho, alocação de recursos, triagem de pacientes, garantia de qualidade de pessoal de enfermagem, entre outras.

Há que se considerar que as condições de trabalho interferem diretamente na carga de trabalho dos profissionais de enfermagem. Os estudos apontam que essas condições, muitas vezes deficientes, necessitam ser minimizadas, porém os autores não as relacionam com as propostas de dimensionamento de pessoal. Ou seja, classifica-se o paciente quanto à necessidade de assistência, sem considerar as condições que se têm para atendê-lo. Isso pode contribuir para que haja em muitos serviços um quantitativo aparentemente insuficiente para prestar uma assistência de qualidade, o que poderia ser revertido com melhores condições de trabalho.

$\mathrm{O}$ enfermeiro tem se preocupado com a responsabilidade de dimensionar o pessoal de enfermagem para seu local de trabalho e buscado caminhos que lhe deem objetividade e suporte teórico para negociar frente às administrações hospitalares. Nesse sentido, necessita-se conhecer a carga de trabalho a que está submetida a equipe de enfermagem, como uma das variáveis importantes para o dimensionamento adequado de pessoal.

\section{Referências}

1. Brasil. Diário Oficial da República Federativa do Brasil. Lei 7498 de 25 de junho de 1986. Dispõe sobre o regulamento e o exercício da enfermagem e dá outras providências. Brasilia, junho de 1986, seção 1.

2. Conselho Federal de Enfermagem. Resolução 293/2004. Fixa e estabelece parâmetros para o dimensionamento do quadro de profissionais de enfermagem nas unidades assistenciais das instituições de saúde e assemelhados. São Paulo: Cofen, 2004.

3. Gaidzinski, R.R., Fugulin, F.M.T., Castilho, V. “Dimensionamento de pessoal de enfermagem em instituições de ensino". In: Kurcgant, P. (coordenador). Gerenciamento em Enfermagem. Rio de Janeiro: Guanabara-Koogan, 2005. 4. Conselho Federal de Enfermagem. Resolução no 189/1996. Estabelece parâmetros para dimensionamento do quadro de profissionais de enfermagem nas instituiçōes de saúde. In: Conselho Regional de Enfermagem de São Paulo. Documentos Básicos de Enfermagem: Enfermeiros, Técnicos, Auxiliares. São Paulo: Coren/SP, 2001. 5. Gaidzinski, R.R. Dimensionamento de Pessoal de Enfermagem em Instituições Hospitalares [livre docência]. São Paulo: Escola de Enfermagem da USP, 1998. 6. Conishi, R.M.Y., Gaidzinski, R.R. “Nursing Activities Score (NAS) como instrumento para medir carga de trabalho de enfermagem em UTI adulto". Rev. Esc. Enferm. USP, 2007, 41(3).

7. Ganong, L.H. "Integrative review of nursing research". Res. Nurs. Health, 1987, 10(1):1-11.

8. Perroca, M.G. Instrumento de Classificação de Pacientes de Perroca:Validação Clínica [tese]. São Paulo: Escola de Enfermagem da USP, 2000.

9. Nunes, B. Tradução para o Português e Validação de um Instrumento de Medida de Gravidade em UTI: Tiss-28 - Therapeutic Intervention Scoring System [dissertação]. São Paulo: Escola de Enfermagem da USP, 2002.

10. Queijo, A.F. Tradução para o Português e Validação de um Instrumento de Medida de Carga de Trabalho de Enfermagem em Unidade de Terapia Intensiva: Nursing Activities Score (NAS) [dissertação]. São Paulo: Escola de Enfermagem da USP, 2002.
11. Ducci, A.J., Zanei, S.S.V., Whitaker, I.Y. "Carga de trabalho de enfermagem para quantificar proporção profissional de enfermagem/paciente em UTI cardiológica". Rev. Esc. Enferm. USP, 2008, 42(4).

12. Sousa, C.R., Gonçalves, L.A., Toffoleto, M.C., Leão, K., Padilha, K.G. "Preditores da demanda de trabalho de enfermagem para idosos internados em unidade de terapia intensiva". Rev. Latinoam. Enferm., 2008, 16(2):218-23.

13. Balsanelli, A.P., Zanei, S.S.V., Whitaker, I.Y. "Carga de trabalho de enfermagem e sua relação com a gravidade dos pacientes cirúrgicos em UTI". Acta Paul. Enferm., 2006, 19(1):16-20.

14. Nogueira, G.P. Indicadores de Gravidade em Unidades de Terapia Intensiva: Estudo Comparativo entre o"Therapeutic Intervention Scoring System-28 e Sua Versão Reduzida" [dissertação]. São Paulo: Escola de Enfermagem da USP, 2002. 15. Santos, E.M., Araújo, T.M. “Processo de trabalho e saúde dos trabalhadores no Hospital Universitário Professor Edgard Santos". Rev. Baiana Saúde Pública, 2003, 27(2):155-68.

16. Sêcco, I.A.O. Acidentes e Cargas de Trabalho dos Trabalhadores de Enfermagem de um Hospital Universitário do Norte do Paraná [tese]. São Paulo: Escola de Enfermagem da USP, 2006.

17. Cheavegatti, D. Trabalho e Adoecimento na Perspectiva de Agentes Comunitários de Saúde da Coordenadoria de Saúde Centro-Oeste do Município de São Paulo [dissertação]. São Paulo: Escola de Enfermagem da USP, 2008. 18. Costa, C.C. Aspectos Ergonômicos na Organização do Trabalho da Equipe de Enfermagem de uma UTI Adulto [dissertação]. Rio Grande do Sul: Universidade Federal do Rio Grande do Sul, 2008.

19. Piesanti, S,R. A Carga de Trabalho dos Auxiliares de Enfermagem de uma Unidade de Terapia Intensiva de um Hospital Neurocirúrgico [dissertação]. Rio Grande do Sul: Universidade Federal do Rio Grande do Sul, 2004. 20. Ribeiro, E.J.G., Shimizu, H.E. "Acidentes de trabalho com trabalhadores de enfermagem". Rev. Bras. Enferm., 2007, 60(5):535-40. 